\title{
Scale model experiment on room acoustics by hybrid simulation technique
}

\author{
Yoshito Hidaka, Hiroo Yano, and Hideki Tachibana \\ Institute of Industrial Science, University of Tokyo, \\ 7-22-1, Roppongi, Minato-ku, Tokyo, 106 Japan
}

(Received 8 December 1988)

\begin{abstract}
As a method to extend the efficiency of scale model experiment of room acoustics, the hybrid simulation technique has been developed, in which impulse responses are measured in a 1/10 scale model auditorium and they are convolved with arbitrary anechoic source signals like music and speech by the digital convolution technique. Through these processes, the "hall tones" can be heard in advance of construction of the real auditorium. A spark discharge sound source with high repeatability and broad band spectra has been developed for the impulse response measurement, and a simple model dummy head was used for binaural recording. As the result of various contrivances for computer programming, considerably fast convolution calculation has been realized.
\end{abstract}

PACS number: 43. 55. Br, 43. 55. Ka, 43. 60. Gk, 43. 60. Qv, 43. 88. Yn

\section{INTRODUCTION}

The behavior of sound waves in a room is too much complicated for numerical analysis, and therefore scale model experiments have been often performed in the field of room acoustics. In 1934, Spandok carried out the first genuine acoustic scale model experiment on auditorium acoustics. ${ }^{1)}$ After that, a lot of investigations have been made to establish the simulation technique. Through these endeavors and with the aid of recent development of measuring devices, it has become possible to make acoustic scale model experiments with considerably high accuracy.

We have also investigated the simulation techniques and various experimental methods so far, ${ }^{2-5}$ ) and have performed scale model experiments for acoustic design of several auditoriums in Japan.

Furthermore, we are now developing a hybrid simulation method to synthesize the sound in model auditorium by using physical scale modeling together with digital signal processing. ${ }^{6}$ ) In this paper, the outline of this simulation technique is reported.

\section{TECHNIQUES FOR $1 / 10$ SCALES MODEL EXPERIMENT}

For scale model experiment in room acoustics, various scale factors are chosen according to the aim of study. Among them, the scale factor of $1 / 10$ is most often used for precise model experiment. In the case of $1 / 10$ scale model experiment, the simulation of boundary condition is relatively easy, ${ }^{3)}$ and the atmospheric sound absorption can be simulated by using dry air or nitrogen gas as the model medium. $^{2)}$

In this kind of model experiments, such physical quantities as reverberation time, sound pressure level distribution, impulse response (echo time pattern), and various psycho-acoustical parameters are measured to predict the room acoustics of full scale auditoriums. In addition to these physical measurements, it is eagerly desired to listen to the "hall tones" in a model auditorium according to the similarity rules of acoustic scale modeling. In order to realize this desire, a lot of trials have been made so far by using tape recorders with changeable tapespeed function and various kinds of loudspeaker 
for high frequency reproduction. ${ }^{4)}$ By doing such experiment, we can hear the sounds with reverberation of the model auditorium. When these analog type instruments are used, however, it is rather difficult to obtain high quality sounds with flat frequency characteristic and sufficient signal to noise $(\mathrm{S} / \mathrm{N})$ ratio.

Instead of such classical techniques, new simulation methods are being developed recently by using physical modeling technique together with digital signal processing technique. That is, the impulse responses from a sound source to receiving points including the room effects are to be measured by scale model experiment, and they are to be convolved with anechoic sound signals like music and speech by using a digital computer. Regarding this technique, $\mathrm{H}$. Els and J. Brauert have already made the first trial by using a loudspeaker and a precise model dummy head system. ${ }^{7}$ We have also investigated a similar technique, and much improved result compared with the classical simulation has been obtained recently. ${ }^{6)}$

\section{HYBRID SIMULATION TECHNIQUE}

As is well known, the physical properties of a linear time-invariant system are described only by its impulse response. Therefore, if the impulse response from a sound source to a receiving point can be exactly measured, we can obtain the output when an arbitrary signal is fed into the acoustic system by the convolution principle.

Although this technique is very simple in theory, there are various problems to be solved when it is actually applied to scale model experiment on room acoustics. Accordingly, we have made some investigations as follows.

\subsection{Scale Model Experiment}

\subsubsection{Impulsive sound source}

For the measurement of impulse response of a room, the most basic and direct method is to use an impulsive sound source with short duration time. In this case, however, it is rather difficult to get sufficient energy to realize high $\mathrm{S} / \mathrm{N}$ ratio. For this reason, other methods have been contrived so far, such as the time-stretch and compression technique $^{8,9)}$ and the cross-correlation technique using pseudo-random noise. ${ }^{10,11)}$ These methods have an advantage that relatively high $\mathrm{S} / \mathrm{N}$ ratio can be realized, while it is necessary to use a transducer like loudspeaker which can reproduce arbitrary signals. In the case of scale model experiment, it is difficult to realize this kind of transducer with sufficiently small size and omnidirectivity.

By considering these points, we decided to adopt the direct method using impulsive sound source and contrived a spark discharge source with refer to literature 12. In the case of this kind of source, to get a waveform with high repeatability and to get a big sound energy are incompatible conditions. We attached greater importance to the former condition. Concerning the latter condition, $\mathrm{S} / \mathrm{N}$ ratio can be improved by the synchronous averaging technique if the waveform has high repeatability.

Figure 1 shows the electrodes of the spark dis-

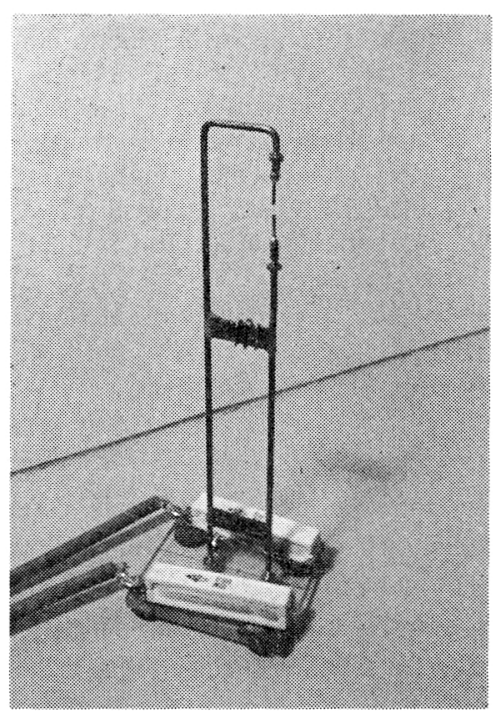

Fig. 1 Electrodes of the spark discharge sound source.

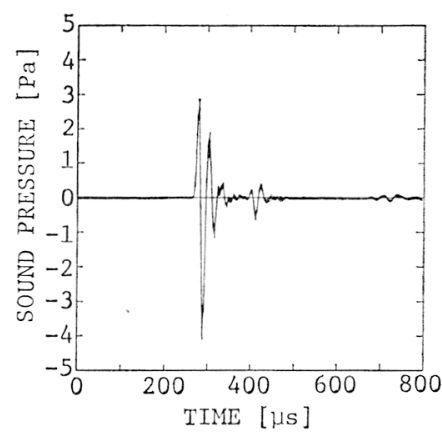

Fig. 2 Sound pressure waveform of the spark discharge sound source (superposed 64 times). 

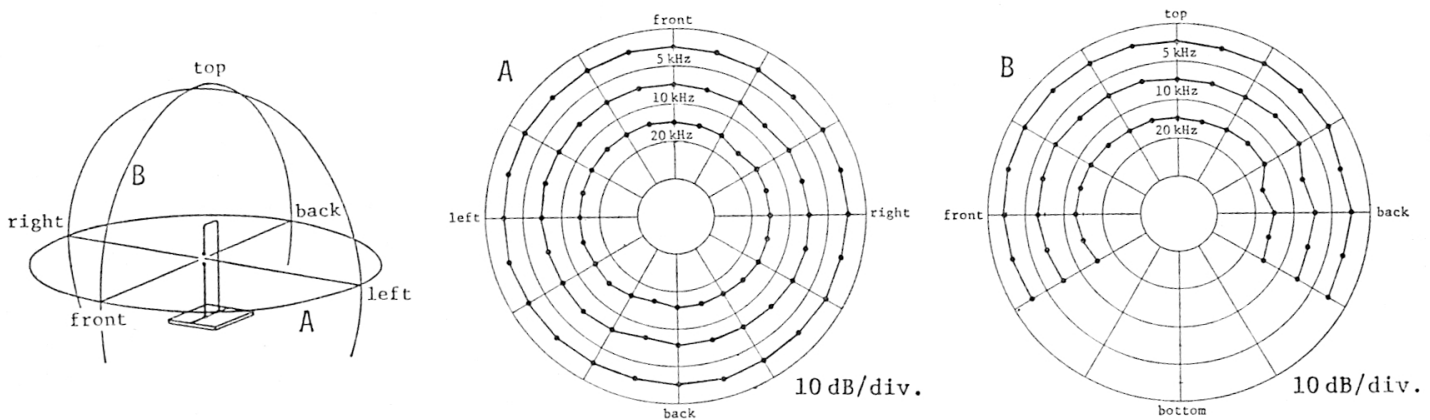

Fig. 3 Directivity characteristic of the spark discharge sound source.

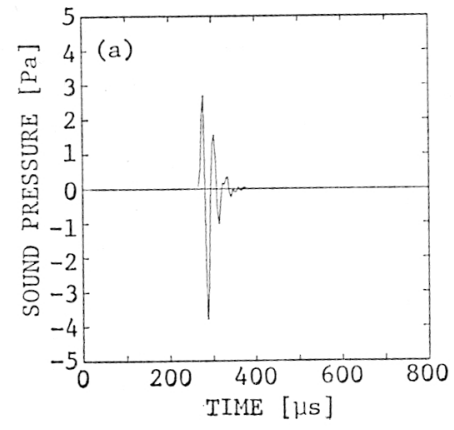

(a)

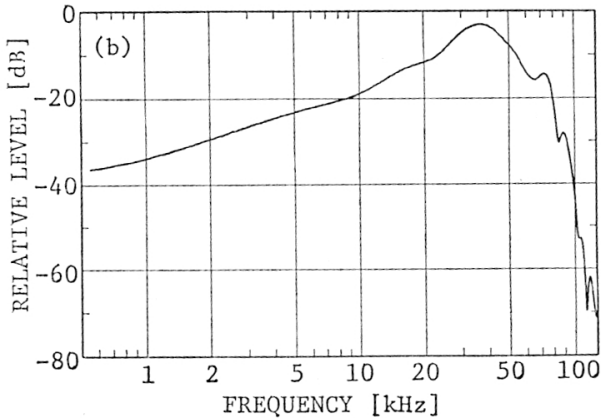

(b)

Fig. 4 Waveform and energy spectrum of the spark discharge sound source. (a) Sound pressure waveform, (b) Energy spectrum.

charge sound source which are in needle to needle configuration with $5 \mathrm{~mm}$ gap. Figure 2 shows the waveforms superposed 64 times of the sound pressure measured at a distance of $50 \mathrm{~cm}$ from the electrodes. In this measurement, a $1 / 4$ in. condenser microphone (B \& K 4135) was used and the cut-off frequency of the anti-aliasing filter was set at $90 \mathrm{kHz}$. As is clearly seen in this figure, a fairly good repeatability has been achieved. This impulsive sound source has a sufficient omnidirectivity as shown in Fig. 3.

These waveforms were averaged synchronously on a personal computer (NEC PC98-XL) and the direct sound was cut from the result. The waveform and energy spectrum of the direct sound are shown in Fig. 4. Although the energy spectrum is not flat, no dips are observed over a wide frequency range. Therefore, the spectral characteristic can be corrected by the inverse filtering technique as mentioned later.

\subsubsection{Receiving system}

In order to realize natural hearing sensation, the

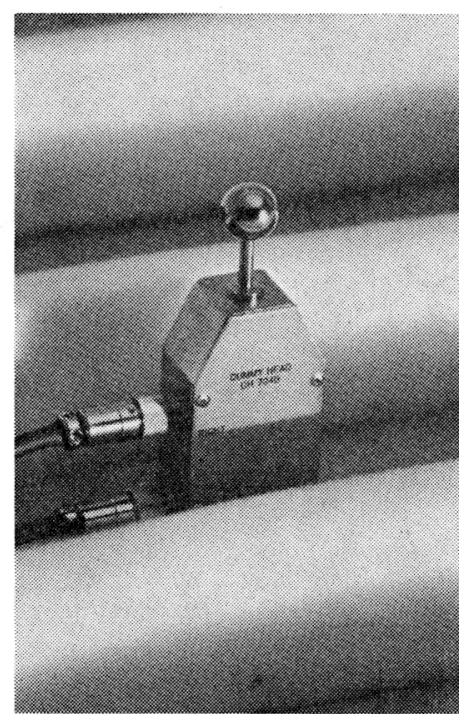

Fig. 5 1/10 scale model dummy head. 
binaural recording technique using a head and torso simulator (dummy head) is often used. This technique seems to be effective also in scale model experiment. So, in 1979, a model dummy head was made on trial base and was preliminarily used in a $1 / 10$ scale model experiment in our laboratory. ${ }^{13)}$ Recently, H. Els and J. Blauert made a much sophisticated model dummy head to simulate the sensation of sound source localization accurately. ${ }^{7)}$

In this study, we made a new model dummy head shown in Fig. 5 and used in 1/10 scale model experiments mentioned below. This model dummy head has a very simple construction consisting of a metal sphere of $15 \mathrm{~mm}$ diameter in which two $1 / 6 \mathrm{in}$. condenser microphones are installed and a metal torso in which two channel preamplifiers are built in. At present, this receiving system is being used without pinnas.

\subsection{Digital Signal Processing}

3.2.1 Correction of spectral characteristic of the sound source

In order to obtain accurate impulse responses in model auditoriums, the deconvolution (inverse filtering) technique was applied as follows.

At first, a linear phase FIR band pass filter of 255 points with frequency range from $1.3 \mathrm{kHz}$ to $80 \mathrm{kHz}$ shown in Fig. 6 was designed. This frequency range was chosen by considering the spectral characteristic of the spark discharge source and the frequencies required for $1 / 10$ scale model experiment. Next, an inverse filter of 512 points was obtained by deconvolution so that it generates the impulse response of the band pass filter when the source impulse is applied. Figure 7 shows the impulse response and the spectral characteristic of the inverse filter. At last, to correct the impulse responses measured in the model by the spark discharge source,

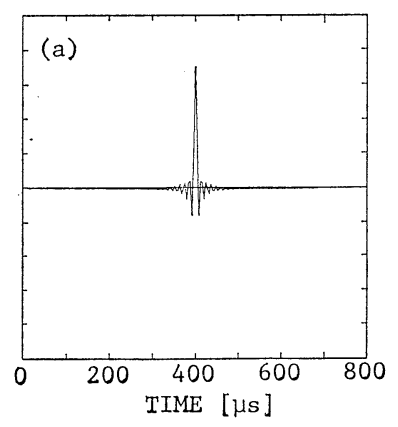

(a)

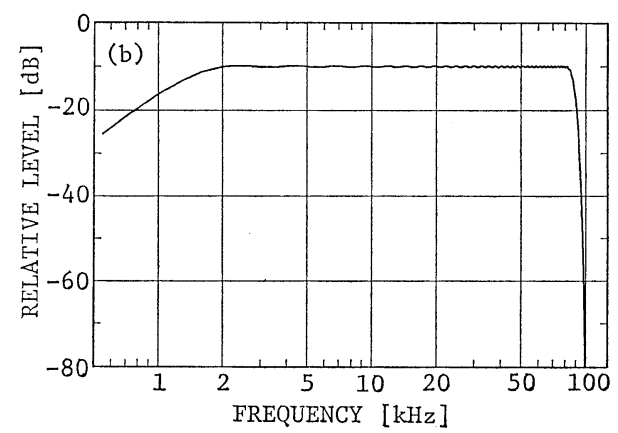

(b)

Fig. 6 Impulse response and the frequency characteristic of a liner phase FIR band pass filter. (a) Impulse response, (b) Frequency characteristic.

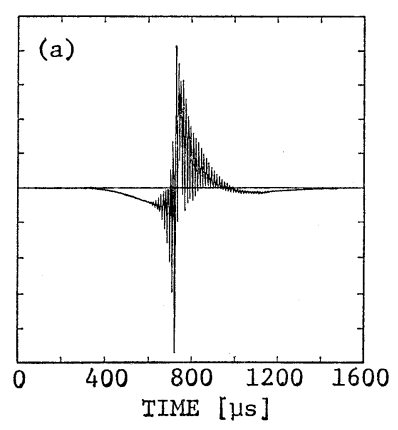

(a)

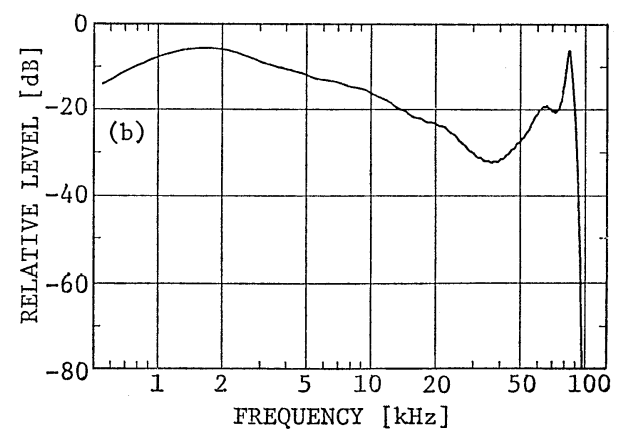

(b)

Fig. 7 Impulse response and frequency characteristic of the inverse filter. (a) Impulse response, (b) Frequency characteristic. 


\section{Y. HIDAKA et al: : HYBRID SIMULATION OF ROOM ACOUSTICS}

they were convolved with the impulse response of the inverse filter.

In the designing of the FIR band pass filter, the calculation program described in literature 14 was used, and the inverse filter was computed by the least-squares method. ${ }^{15)}$

3.2.2 Convolution of impulse response and source signal

The impulse responses measured in a model auditorium through the model dummy head are to be corrected in the manner mentioned above and then, they are to be convolved with an arbitrary source signal.

Since the convolution of two long signals is much time-consuming, it was performed on a host computer by using the overlap-add method. ${ }^{16)}$ In this method, the source signal to be convolved is split into a lot of pieces of time function so that the fast Fourier transform for each piece can be easily computed. The length of each piece was chosen as the same as the length of the impulse responses.

In these computation programs, the following techniques were adopted to improve the efficiency of computation ${ }^{17)}$ : (1) to look up a tabulated trigonometric function, (2) to use a radix 8 routine for real data, (3) to save the processing of bit-reversal reordering by use of the decimation-in-frequency algorism for forward FFT and the decimation-intime algorism for inverse FFT.

\subsection{Experimental System}

Figure 8 shows the block diagram of our experimental system. The measurement of impulse response is controlled by a personal computer (NEC PC98-XL), in which the data acquisition through a 12-bit A/D converter (ELMEC EC-2390) and the synchronous averaging are carried out automatically. In the impulse response measurement, $2^{16}$ samples per a channel are obtained at a sampling frequency of $320 \mathrm{kHz}$, which approximately correspond to the length of reverberation time in a model auditorium. Figure 9 shows an example of a pair of impulse responses (right and left channels) measured in a model auditorium through the model dummy head. In this measurement, the synchronous averaging was done 320 times.

The 16-bit digital data of the source signal recorded on a digital-autio-taperecorder (DAT) are transferred to the personal computer through a DATinterface (MTT DAI-98B). Next, these data of the impulse responses and the source signal are transferred to a host computer (FACOM M-380Q) via floppy disks, and the inverse filtering for the correction of the spectral characteristic of the impulsive

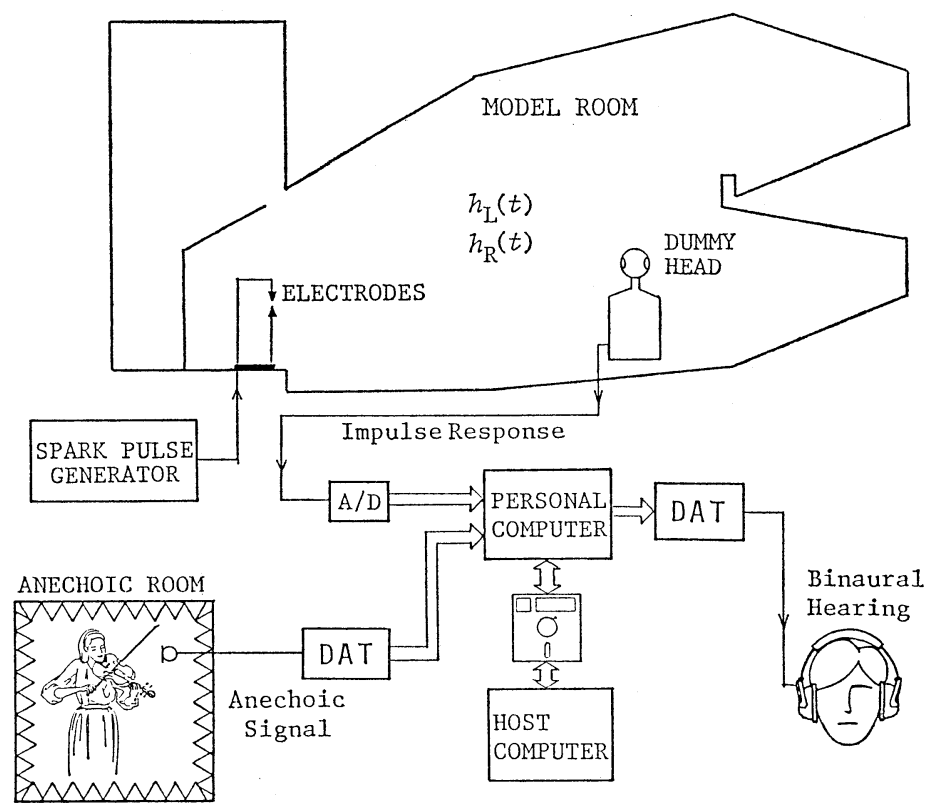

Fig. 8 Acoustic scale model experiment by the hybrid simulation technique. 


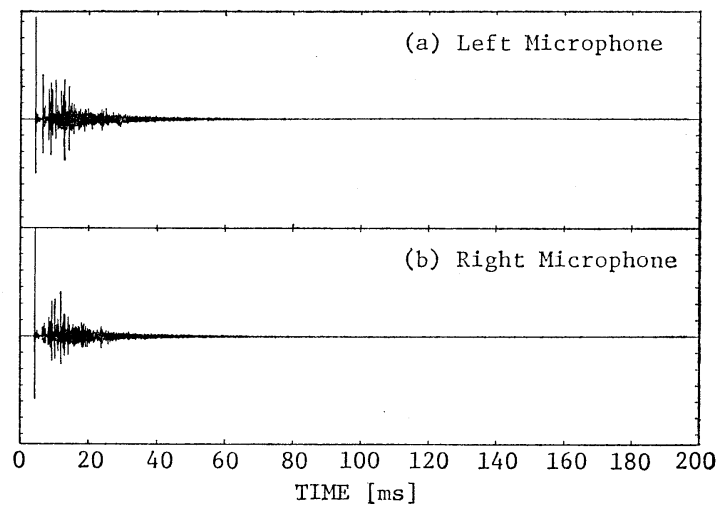

Fig. 9 A pair of impulse responses measured in a $1 / 10$ scale model auditorium through the model dummy head.

source and the convolution calculation were performed there. As the result of the contrivances mentioned above, the calculation time has become considerably short. For instance, the convolution of a source signal of about 30 seconds and two channel impulse responses of a model concert hall can be performed in about 120 seconds.

The results of the computation are again transferred back to the personal computer and then recorded on the DAT. Finally, by reproducing the 2-channel recordings through headphones, we can hear the sound with reverberation in the model auditorium.

\section{TRIALS IN 1/10 SCALE MODEL EXPERIMENTS OF AUDITORIUMS}

Several concert halls and theaters are now under designing in Japan. Among them, the authors are taking part in the acoustic design works for the Second National Theater in which an opera theater of 1,800 seats and a multi-purpose theater of 950 seats are contained and the Aichi Prefectural Culture Center in which a concert hall of 1,800 seats and a theater of 2,500 seats are contained. In order to make detailed acoustical examinations in these works, $1 / 10$ scale model experiments of these four auditoriums were performed, in which the hybrid simulation technique mentioned above was applied on trial.

In these experiments, the impulse responses between a representative source position on the stage and a lot of receiving positions in the audience area were measured by using the spark discharge sound source and the model dummy head. Subsequently, these results were convolved with various source signals such as solo musics (a violin, a flute and four opera singers) and a string quartet recorder under anechoic condition. These synthesized sounds were presented to listners through headphones.

Although genuine subjective tests have not yet been performed, preliminary presentations were made to acousticians, architects and musicians. As a result, it has been found that, at first, the sound quality has been much improved compared with the classical simulation, and subjective differences of such sensations as loudness and reverberancy can be clearly judged and spatial impression has been realized to some extent.

Beside the synthesized musics, the impulse responses were also presented to the listeners. Of course, their frequencies were converted into audio frequency range. As a result, it turned out that the binaural impulse responses are proper test signals for the judgement of acoustic conditions, especially when they are judged by much experienced acousticians.

In the preliminary experiments mentioned above, point to point impulse responses were measured by supposing a point source like solo music. At the next stage, impulse responses from several points to a receiving point are to be measured and they are to be convolved with multi-channel source signals. By doing so, sound sources with spatial extent like orchestra and opera performances will be simulated.

\section{CONCLUSIONS}

In this paper, some technical investigations regarding the hybrid simulation method for room acoustics have been introduced. From the results of the preliminary experiments, it can be concluded that this kind of simulation technique provides comprehensive design aid for auditoriums, because the "hall tones" can be tested by direct hearing in advance of construction of auditoriums.

However, the present technique is not yet perfect, and there remains some problems to be improved. Among them, the most serious problem is how to precisely measure the impulse response with high $\mathrm{S} / \mathrm{N}$ ratio. If any noise remains in the impulse response, the convolution is to made with it and consequently false "reverberancy" is attached. As 


\section{Y. HIDAKA et al.: HYBRID SIMULATION OF ROOM ACOUSTICS}

the receiving system, a very simple model dummy head is being used in our present work. It should be improved so as to realize sufficient binaural sensation.

In the simulation technique mentioned in this paper, $1 / 10$ scale models are used to predict the impulse responses of auditoriums. However, it is desirable to obtain them by numerical calculation. In order to realize such a perfect digital simulation technique, it is necessary to develop a precise method of calculating the impulse responses including the wave phenomenon.

\section{REFERENCES}

1) F. Spandok, "Raumakustisch Modellversuche," Ann. Phys. V 20, 345-360 (1934).

2) H. Tachibana, K. Ishii, and O. Hirano, " $\mathrm{N}_{2}$-substitution method for acoustic scale model experiment," J. Acoust. Soc. Jpn. (J) 27, 163-169 (1971) (in Japanese).

3) H. Tachibana and K. Ishii, "Simulation of the sound absorption characteristics of the absorbents for acoustic scale model experiment," J. Acoust. Soc. Jpn. (J) 28, 169-175 (1972) (in Japanese).

4) H. Tachibana and K. Ishii, "Similarity rules in acoustic scale model study and experimental techniques," J. Acoust. Soc. Jpn. (J) 32, 621-630 (1976) (in Japanese).

5) H. Tachibana, H. Yano, and Y. Hidaka, "Scale model experiment on room acoustics," Proc. Int. Symp. Scale Modeling, 181-187 (1988).

6) Y. Hidaka, H. Yano, and H. Tachibana, "Basic study on hybrid acoustic scale model experiments," Proc. Spring Meet. Acoust. Soc. Jpn., 561-562 (1988) (in Japanese).

7) H. Els and J. Blauert, "A measuring system for acoustic scale models," Proc. Vancouver Symp.
Acoustics and Theatre Planning for the Performing Arts, 65-70 (1986).

8) A. J. Berkhout, D. de Vries, and M. M. Boone, "A new method to acquire impulse responses in concert halls," J. Acoust. Soc. Am. 68, 179-183 (1980).

9) N. Aoshima, "Computer-generated pulse signal applied for sound measurement," J. Acoust. Soc. Am. 69, 1484-1488 (1981).

10) M. R. Schroeder, "Integrated-impulse method measuring sound decay without using impulses," J. Acoust. Soc. Am. 66, 497-500 (1979).

11) H. Alrutz and M. R. Schroeder, "A Fast Hadamard Transform method for the evaluation of measurements using pseudorandom test signals," Proc. 11th ICA, 235-238 (1983).

12) H. Shibayama, K. Fukunaga, and K. Kido, "A proposition of a pulse discharge sound source for acoustical measurements," J. Acoust. Soc. Jpn. (J) 40, 152-160 (1984) (in Japanese).

13) Y. Yamamoto, H. Tachibana, and K. Ishii, "Preliminary study on dummy head for $1 / 10$ scale model experiment," Proc. Autumn Meet. Acoust. Soc. Jpn., 447-448 (1979) (in Japanese).

14) J. H. McClellan, T. W. Parks, and L. R. Rabiner, "A computer program for designing optimum FIR linear phase digital filters," IEEE Trans. AU-21, 506-526 (1973).

15) J. Kawaura, Y. Suzuki, T. Sone, and J. Soma, “On the method of simulating an acoustic transmission system by use of digital signal processing," $\mathbf{J}$. Acoust. Soc. Jpn. (J) 42, 774-779 (1986) (in Japanese).

16) A. V. Oppenheim and R. W. Schafer (trans. by H. Date), Digital Signal Processing, Vol. 1 (CORONA, Tokyo, 1978), Chap. 3.

17) A. V. Oppenheim and R. W. Schafer (trans. by H. Date), Digital Signal Processing, Vol. 1 (CORONA, Tokyo, 1978), Chap. 6. 